\title{
Tree-level lepton universality violation in the presence of sterile neutrinos: impact for $R_{K}$ and $R_{\pi}$
}

\author{
A. Abada, ${ }^{a}$ D. Das, ${ }^{a}$ A.M. Teixeira, ${ }^{b}$ A. Vicente ${ }^{a}$ and C. Weiland ${ }^{a}$ \\ ${ }^{a}$ Laboratoire de Physique Théorique, CNRS - UMR 8627, Université de Paris-Sud 11, \\ F-91405 Orsay Cedex, France \\ ${ }^{b}$ Laboratoire de Physique Corpusculaire, CNRS/IN2P3 - UMR 6533, \\ Campus des Cézeaux, 24 Av. des Landais, F-63171 Aubière Cedex, France \\ E-mail: abada@th.u-psud.fr, debottam.das@th.u-psud.fr, \\ ana.teixeira@clermont.in2p3.fr, avelino.vicente@th.u-psud.fr, \\ cedric.weiland@th.u-psud.fr
}

ABStract: We consider a tree-level enhancement to the violation of lepton flavour universality in light meson decays arising from modified $W \ell \nu$ couplings in the standard model minimally extended by sterile neutrinos. Due to the presence of additional mixings between the active (left-handed) neutrinos and the new sterile states, the deviation from unitarity of the leptonic mixing matrix intervening in charged currents might lead to a tree-level enhancement of $R_{P}=\Gamma(P \rightarrow e \nu) / \Gamma(P \rightarrow \mu \nu)$, with $P=K, \pi$. We illustrate these enhancements in the case of the inverse seesaw model, showing that one can saturate the current experimental bounds on $\Delta r_{K}$ (and $\Delta r_{\pi}$ ), while in agreement with the different experimental and observational constraints.

Keywords: Rare Decays, Beyond Standard Model, Neutrino Physics, Kaon Physics

ARXIV EPRINT: 1211.3052 


\section{Contents}

1 Introduction 1

$2 \Delta r_{K}$ in the presence of sterile neutrinos 3

3 Constraints on sterile neutrinos 5

$4 \Delta r_{K}$ in the inverse seesaw model $\quad 6$

4.1 The inverse seesaw model 6

$\begin{array}{lll}4.2 & \text { Numerical evaluation of } \Delta r_{K} \text { in the inverse seesaw model } & 7\end{array}$

$\begin{array}{llr}5 & \text { Concluding remarks } & 9\end{array}$

\section{Introduction}

Lepton flavour universality (LFU) is one of the distinctive features of the Standard Model of strong and electroweak interactions (SM); hence, any deviation from the expected SM theoretical estimates in electroweak precision tests will signal the presence of New Physics (NP). Here we focus on light meson ( $K$ and $\pi$ ) leptonic decays which, in view of the expected experimental precision, have a unique potential to probe deviations from the SM regarding lepton universality.

In the SM, the dominant contribution to $\Gamma(P \rightarrow \ell \nu)(P=K, \pi)$ arises from $W$ boson mediated exchanges. The prediction of each specific decay is heavily plagued by hadronic matrix element uncertainties; however, by considering the ratios

$$
R_{K} \equiv \frac{\Gamma\left(K^{+} \rightarrow e^{+} \nu\right)}{\Gamma\left(K^{+} \rightarrow \mu^{+} \nu\right)}, \quad R_{\pi} \equiv \frac{\Gamma\left(\pi^{+} \rightarrow e^{+} \nu\right)}{\Gamma\left(\pi^{+} \rightarrow \mu^{+} \nu\right)}
$$

the hadronic uncertainties cancel out to a good approximation, so that the SM predictions can be computed with a high precision. In order to compare the experimental bounds with the SM predictions, it proves convenient to introduce a quantity, $\Delta r_{P}$, which parametrizes deviations from the SM expectations, possibly arising from NP contributions:

$$
R_{P}^{\exp }=R_{P}^{\mathrm{SM}}\left(1+\Delta r_{P}\right) \quad \text { or equivalently } \quad \Delta r_{P} \equiv \frac{R_{P}^{\exp }}{R_{P}^{\mathrm{SM}}}-1
$$

The comparison of theoretical analyses $[1,2]$ with the recent measurements from the NA62 collaboration [3] and with the existing measurements on pion leptonic decays [4]

$$
\begin{array}{ll}
R_{K}^{\mathrm{SM}}=(2.477 \pm 0.001) \times 10^{-5}, & R_{K}^{\exp }=(2.488 \pm 0.010) \times 10^{-5} \\
R_{\pi}^{\mathrm{SM}}=(1.2354 \pm 0.0002) \times 10^{-4}, & R_{\pi}^{\exp }=(1.230 \pm 0.004) \times 10^{-4}
\end{array}
$$


suggests that observation agrees at $1 \sigma$ level with the SM's predictions for

$$
\Delta r_{K}=(4 \pm 4) \times 10^{-3}, \quad \Delta r_{\pi}=(-4 \pm 3) \times 10^{-3} .
$$

The current experimental uncertainty in $\Delta r_{K}$ (of around $0.4 \%$ ) should be further reduced in the near future, as one expects to have $\delta R_{K} / R_{K} \sim 0.1 \%$ [5], which can translate into measuring deviations $\Delta r_{K} \sim \mathcal{O}\left(10^{-3}\right)$. There are also plans for a more precise determination of $\Delta r_{\pi}[6,7]$.

Whether or not $R_{P}$ can probe a model of NP naturally depends on the nature and on the expected size of the corresponding contributions to $\Delta r_{P}$. LFU in light meson decays can be violated due to (i) a new Lorentz structure in the four-fermion interaction (arising from the exchange of new fields); (ii) corrections to the SM charged current interaction $W \ell \nu$ vertex. The first possibility has been extensively discussed in the literature, especially in the framework of models with an enlarged Higgs sector, since in the presence of charged scalar Higgs, new tree-level contributions are expected. However, as in the case of most of Two Higgs Doublet Models (2HDM) ${ }^{1}$ or supersymmetric (SUSY) extensions of the SM, these new tree-level corrections are lepton universal [9]. In SUSY models, higher order non-holomorphic couplings can indeed provide new contributions to $R_{P}$ [10-14], but in view of current experimental bounds (collider, $B$-physics and $\tau$-lepton decays), one can have at most $\Delta r_{K} \leq 10^{-3}$ in the framework of unconstrained minimal SUSY models [14]. Corrections to the $W \ell \nu$ vertex (case ii) can also induce violation of LFU in charged currents. Loop level corrections to the latter vertex have been considered and, as referred to in [11], new particles (at a scale $\sim \Lambda_{\mathrm{NP}}$ ) can indeed account for such a contribution, but the effect is of order $(\alpha / 4 \pi) \times\left(m_{W}^{2} / \Lambda_{\mathrm{NP}}^{2}\right)$ and generally well below experimental sensitivity.

The tree-level corrections to charged current interactions, once neutrino oscillations are incorporated into the SM, are more interesting. In this case, and working in the basis where the charged lepton mass matrix is diagonal, the flavour-conserving term $\propto g \bar{l}_{j} \gamma^{\mu} P_{L} \nu_{j} W_{\mu}^{-}$ now reads

$$
-\mathcal{L}_{c c}=\frac{g}{\sqrt{2}} U_{\nu}^{j i} \bar{l}_{j} \gamma^{\mu} P_{L} \nu_{i} W_{\mu}^{-}+\text {c.c. },
$$

where $U_{\nu}^{j i}$ is a generic leptonic mixing matrix, $i=1, \ldots, n_{\nu}$ denoting the physical neutrino states (not necessarily corresponding to the three left-handed SM states $\equiv \nu_{L}$ ) and $j=1, \ldots, 3$ the charged lepton flavour. In the case of three neutrino generations, $U_{\nu}^{j i}$ corresponds to the unitary PMNS matrix and flavour universality is preserved in meson decays: since one cannot tag the flavour of the final state neutrino (missing energy), the meson decay amplitude is proportional to $\left(U_{\nu} U_{\nu}^{\dagger}\right)_{j j}=1$, and thus no new contribution to $R_{P}$ is expected.

In order to account for neutrino masses and mixings, the SM can be extended with new neutral sterile fermion states $\left(n_{\nu}>3\right)$. In many neutrino mass models there are additional singlet states; the existence of sterile states is also strongly supported by current data from reactor experiments, cosmology, as well as indications from large structure formation [15].

In the presence of sterile states, the $W \ell \nu$ vertex is proportional to a rectangular $3 \times n_{\nu}$ matrix $U_{\nu}^{j i}$, and the mixing between the left-handed leptons $\nu_{L}, \ell_{L}$ corresponds to a $3 \times 3$

\footnotetext{
${ }^{1}$ As recently shown in [8], the conclusions can be different in a more generalised 2HDM framework.
} 
block of $U_{\nu}^{j i}$,

$$
U_{\mathrm{PMNS}} \rightarrow \tilde{U}_{\mathrm{PMNS}}=(\mathbb{1}-\eta) U_{\mathrm{PMNS}} .
$$

The larger the mixing between the active (left-handed) neutrinos and the new states, the more pronounced the deviations from unitarity of $\tilde{U}_{\text {PMNS }}$, parametrized by the matrix $\eta[16,17]$. The active-sterile mixings and the departure from unitarity of $\tilde{U}_{\text {PMNS }}$ can be at the source of the violation of LFU in different neutrino mass models which introduce sterile fermionic states (gauge singlets, not necessarily right-handed) to generate non-zero masses and mixings for the light neutrinos. If these new states have very small mixings with the left-handed neutrinos, then $\tilde{U}_{\mathrm{PMNS}} \approx U_{\mathrm{PMNS}}$ and no effect is expected regarding LFU violation (for instance in the case of high-scale type I and III fermionic seesaws). On the other hand, if the model is such that the singlet states are not excessively heavy and have large mixings with the active ones, then the deviations from unitarity (i.e., $\eta$ ) can indeed lead to a (tree-level) correction of the $W \ell \nu$ vertex. The impact of the non-unitarity of the lepton mixing matrix on leptonic light meson decays was first investigated in $[18,19]$, prior to the confirmation of neutrino oscillations. In this work, we revisit this idea in the light of recent neutrino data and in view of the present (and future) experimental sensitivities to $\Delta r_{P}[3,5]$.

Corrections to the $W \ell \nu$ vertex can arise in several scenarios with additional (light) singlet states, as is the case of $\nu \mathrm{SM}$ [20], low-scale type-I seesaw [21] and the Inverse Seesaw (ISS) [22], among other possibilities. As we proceed to discuss, should the masses of the new singlet states $\left(\nu_{s}\right)$ be light enough (lighter than the decaying meson), or if the large mixings induce significant unitarity violation ${ }^{2}$ (even for $m_{\nu_{s}} \gg m_{P}$ ), then one might have sizeable $\Delta r_{P}$, within reach of current experimental sensitivities.

In the following section 2 we compute, in a model-independent approach, $\Delta r_{P}$ in the presence of additional fermionic sterile states; we then briefly review in section 3 the most important experimental and observational constraints on the mass of the additional singlet states. In section 4 , we consider the case of the inverse seesaw model to illustrate the impact of sterile neutrinos on $\Delta r_{P}$. Our concluding remarks are summarised in section 5 .

\section{$2 \Delta r_{K}$ in the presence of sterile neutrinos}

Let us consider the SM extended by $N_{s}$ additional sterile states, and conduct a general formulation of leptonic light meson decays. The matrix element for the meson decay $P \rightarrow l_{j} \nu_{i}$ has the generic form

$$
\mathcal{M}_{i j}=\bar{u}_{\nu_{i}}\left(\mathcal{A}^{i j} P_{R}+\mathcal{B}^{i j} P_{L}\right) v_{l_{j}}
$$

no sum implied over the indices of the outgoing leptons $i, j$. Notice that now one has $i=1, \ldots, 3+N_{s}$. The expressions for $\mathcal{A}$ and $\mathcal{B}$ can be read from the effective hamiltonian (obtained after integrating out the $W$ boson in eq. (1.6)),

$$
\mathcal{H}_{c c}=\frac{4 G_{F}}{\sqrt{2}} V_{\mathrm{CKM}}^{q q^{\prime}}\left(U_{\nu}^{j i} \bar{q} \gamma_{\mu} P_{L} q^{\prime} \bar{l}_{j} \gamma^{\mu} P_{L} \nu_{i}\right)
$$

\footnotetext{
${ }^{2}$ For other phenomenological consequences of non-unitarity in the lepton mixing matrix, see ref. [23].
} 
implying that in this framework one has

$$
\begin{aligned}
& (\mathcal{A})^{i j}=\left(\mathcal{A}^{W}\right)^{i j}=-4 G_{F} V_{\mathrm{CKM}}^{u s} f_{P} U_{\nu}^{j i *} m_{l_{j}} \\
& (\mathcal{B})^{i j}=\left(\mathcal{B}^{W}\right)^{i j}=4 G_{F} V_{\mathrm{CKM}}^{u s} f_{P} U_{\nu}^{j i *} m_{\nu_{i}},
\end{aligned}
$$

where $f_{P}$ denotes the meson decay constant and $m_{l_{j}, \nu_{i}}$ the mass of the outgoing leptons.

The expression for $R_{P}$ is finally given by

$$
\begin{aligned}
& R_{P}=\frac{\sum_{i} F^{i 1} G^{i 1}}{\sum_{k} F^{k 2} G^{k 2}}, \quad \text { with } \\
& F^{i j}=\left|U_{\nu}^{j i}\right|^{2} \text { and } G^{i j}=\left[m_{P}^{2}\left(m_{\nu_{i}}^{2}+m_{l_{j}}^{2}\right)-\left(m_{\nu_{i}}^{2}-m_{l_{j}}^{2}\right)^{2}\right]\left[\left(m_{P}^{2}-m_{l_{j}}^{2}-m_{\nu_{i}}^{2}\right)^{2}-4 m_{l_{j}}^{2} m_{\nu_{i}}^{2}\right]^{1 / 2} .
\end{aligned}
$$

The result of eq. (2.5) has a straightforward interpretation: $F^{i j}$ represents the impact of new interactions (absent in the SM), whereas $G^{i j}$ encodes the mass-dependent factors. Notice however that all states (charged and neutral fermions) do not necessarily contribute to $R_{P}$ : this can be seen from inspection of $G^{i j}$, which must be a positive definite quantity. In particular, we denote by $N_{\max }^{\left(l_{j}\right)}$ the $N^{\text {th }}$ heaviest neutrino mass eigenstate which is kinematically allowed.

The SM result can be easily recovered from eq. (2.5), in the limit $m_{\nu_{i}}=0$ and $U_{\nu}^{j i}=\delta_{j i}$,

$$
R_{P}^{S M}=\frac{m_{e}^{2}}{m_{\mu}^{2}} \frac{\left(m_{P}^{2}-m_{e}^{2}\right)^{2}}{\left(m_{P}^{2}-m_{\mu}^{2}\right)^{2}},
$$

to which small electromagnetic corrections (accounting for internal bremsstrahlung and structure-dependent effects) should be added [1].

The general expression for $\Delta r_{P}$ now reads

$$
\Delta r_{P}=\frac{m_{\mu}^{2}\left(m_{P}^{2}-m_{\mu}^{2}\right)^{2}}{m_{e}^{2}\left(m_{P}^{2}-m_{e}^{2}\right)^{2}} \frac{\sum_{m=1}^{N_{\max }^{(e)}} F^{m 1} G^{m 1}}{\sum_{n=1}^{N_{\max }^{(\mu)}} F^{m 2} G^{n 2}}-1 .
$$

Thus, depending on the masses of the new states (and their hierarchy) and most importantly, on their mixings to the active neutrinos, $\Delta r_{P}$ can considerably deviate from zero. In order to illustrate this, we consider two regimes: in the first (A), all sterile neutrinos are lighter than the decaying meson, but heavier than the active neutrino states, i.e. $m_{\nu}^{\text {active }} \ll$ $m_{\nu_{s}} \lesssim m_{P}$; in the second (B), all $\nu_{s}$ are heavier than $m_{P}$. Notice that in case (A), all the mass eigenstates can be kinematically available and one should sum over all $3+N_{s}$ states; furthermore there is an enhancement to $\Delta r_{P}$ arising from phase space factors, see eq. (2.6).

We further emphasise that scenarios (A) and (B) are in general experimentally indistinguishable concerning lepton flavour universality, the only exception corresponding to a very particular regime where the sterile neutrinos are very close in mass to the decaying pseudoscalar meson. ${ }^{3}$

\footnotetext{
${ }^{3}$ In such a situation, the resulting charged lepton would either be less energetic and not pass the experimental kinematical cuts $[18,19]$, or then have a clearly reduced momentum.
} 


\section{Constraints on sterile neutrinos}

There are strong experimental and observational bounds on the mass regimes and on the size of the active-sterile mixings that must be satisfied. Firstly, it is clear that present data on neutrino masses and mixings [24] should be accounted for. Secondly there are robust laboratory bounds from direct sterile neutrinos searches [15, 25], since the latter can be produced in meson decays such as $\pi^{ \pm} \rightarrow \mu^{ \pm} \nu$, with rates dependent on their mixing with the active neutrinos. Negative searches for monochromatic lines in the muon spectrum can be translated into bounds for $m_{\nu_{s}}-\theta_{i \alpha}$ combinations, where $\theta_{i \alpha}$ parametrizes the active-sterile mixing. The non-unitarity of the leptonic mixing matrix is also subject to constraints: the rates for leptonic and hadronic processes with final state neutrinos depend on $\sum_{i}\left|U_{\nu}^{j i}\right|^{2}$, where (as mentioned above) the sum extends over all neutrino states kinematically accessible $\left(i=1, \ldots, N_{\max }\right)$, and thus constrain the departure from the unitarity limit $\sum_{i}\left|U_{\nu}^{j i}\right|^{2}=1$. Bounds on the non-unitarity parameter $\eta$ (eq. (1.7)), were derived using Non-Standard Interactions [26]; although not relevant in case (A), these bounds will be taken into account when evaluating scenario (B).

Unless the active-sterile mixings are negligible, the modified $W \ell \nu$ vertex may also contribute to lepton flavour violation (LFV) processes, ${ }^{4}$ with potentially large rates. $\mu \rightarrow e \gamma$ decays, searched for by the MEG experiment [28], are the most stringent ones ${ }^{5}$ - the rate induced by sterile neutrinos must satisfy $[30,31]$

$$
\operatorname{BR}(\mu \rightarrow e \gamma)=\frac{\alpha_{W}^{3} s_{W}^{2} m_{\mu}^{5}}{256 \pi^{2} m_{W}^{4} \Gamma_{\mu}}\left|H_{\mu e}\right|^{2} \leq 2.4 \times 10^{-12},
$$

where $H_{\mu e}=\sum_{i} U_{\nu}^{2 i} U_{\nu}^{1 i *} G_{\gamma}\left(\frac{m_{\nu, i+3}^{2}}{m_{W}^{2}}\right)$, with $G_{\gamma}$ the loop function and $U_{\nu}$ the mixing matrix defined in eq. (1.6). Similarly, any change in the $W \ell \nu$ vertex will also affect other leptonic meson decays, in particular $B \rightarrow \ell \nu$; the following bounds were enforced in the analysis: $\mathrm{BR}(B \rightarrow e \nu)<9.8 \times 10^{-7}, \mathrm{BR}(B \rightarrow \mu \nu)<10^{-6}$ and $\mathrm{BR}(B \rightarrow \tau \nu)=$ $(1.65 \pm 0.34) \times 10^{-4}[32]$.

Important constraints can also be derived from LHC Higgs searches [33] and electroweak precision data [34]. LHC data on Higgs decays already provides some important bounds when the sterile states are slightly below $125 \mathrm{GeV}$ (due to the potential $H$-decays to left- and right-handed neutrinos). The active-sterile mixings can introduce small deviations to the electroweak fits, which allows to constrain them. An effective approach was applied in [34], assuming very heavy sterile neutrinos, and thus these bounds will only be applied in scenario (B).

Under the assumption of a standard cosmology, the most constraining bounds on sterile neutrinos stem from a wide variety of cosmological observations [15, 35]. Using Large

\footnotetext{
${ }^{4} \mathrm{LFV}$ is typically dipole dominated when the sterile neutrinos are light $\left(m_{\nu_{s}} \lesssim 300 \mathrm{GeV}\right)$, so that $\mu \rightarrow e \gamma$ is the most constraining LFV observable. For heavier sterile neutrinos, other (model-dependent) contributions beyond the dipole might be more relevant [27].

${ }^{5}$ Recently, it has been also noticed that in the framework of a low-scale type I seesaw, the expected future sensitivity of $\mu-e$ conversion experiments can also play a relevant rôle in detecting or constraining sterile neutrino scenarios in the $2 \mathrm{GeV}-1000 \mathrm{TeV}$ mass range [29].
} 
Scale Structure (LSS) data, one can also set relevant bounds on very light sterile neutrinos $\left(m_{\nu_{s}}<100 \mathrm{eV}\right)$, since if such light states constitute a non-negligible fraction of the dark matter of the Universe, then structure formation is affected. Active-sterile mixing also induces radiative decays $\nu_{i} \rightarrow \nu_{j} \gamma$, well constrained by cosmic X-ray searches. Lyman- $\alpha$ limits, the existence of additional degrees of freedom at the epoch of Big Bang Nucleosynthesis, and Cosmic Microwave Background (CMB) data, also allow to set additional bounds in the $m_{\nu_{s}}-\theta_{i \alpha}$ plane. However, all the above cosmological bounds can be evaded if a non-standard cosmology is considered. In fact, the above cosmological constraints disappear in scenarios with a low reheating temperature [36]. In our numerical analysis we will allow for the violation of the latter bounds, explicitly stating it.

\section{$4 \Delta r_{K}$ in the inverse seesaw model}

Although the generic idea explored in this work applies to any model where the active neutrinos have sizeable mixings with some additional singlet states, we consider the case of the Inverse Seesaw [22] to illustrate the potential of a model with sterile neutrinos regarding tree-level contributions to light meson decays. As mentioned before, there are other possibilities [20, 21].

\subsection{The inverse seesaw model}

In the ISS, the SM particle content is extended by $n_{R}$ generations of right-handed $(\mathrm{RH})$ neutrinos $\nu_{R}$ and $n_{X}$ generations of singlet fermions $X$ with lepton number $L=-1$ and $L=+1$, respectively [22] ( $\operatorname{such}$ that $n_{R}+n_{X}=N_{s}$ ). Even if deviation from unitarity can occur for different values of $n_{R}$ and $n_{X}$, here we will consider the case $n_{R}=n_{X}=3$. The lagrangian is given by

$$
\mathcal{L}_{\mathrm{ISS}}=\mathcal{L}_{S M}+Y_{\nu}^{i j} \bar{\nu}_{R i} L_{j} \tilde{H}+M_{R i j} \bar{\nu}_{R i} X_{j}+\frac{1}{2} \mu_{X_{i j}} \bar{X}_{i}^{c} X_{j}+\text { h.c. }
$$

where $i, j=1,2,3$ are generation indices and $\tilde{H}=i \sigma_{2} H^{*}$. Notice that the present lepton number assignment, together with $L=+1$ for the SM lepton doublet, implies that the "Dirac"-type right-handed neutrino mass term $M_{R_{i j}}$ conserves lepton number, while the "Majorana" mass term $\mu_{X_{i j}}$ violates it by two units.

The non-trivial structure of the neutrino Yukawa couplings $Y_{\nu}$ implies that the lefthanded neutrinos mix with the RH ones after electroweak symmetry breaking. In the $\left\{\nu_{L}, \nu_{R}^{c}, X\right\}$ basis, one has the following symmetric $(9 \times 9)$ mass matrix $\mathcal{M}$,

$$
\mathcal{M}=\left(\begin{array}{ccc}
0 & m_{D}^{T} & 0 \\
m_{D} & 0 & M_{R} \\
0 & M_{R}^{T} & \mu_{X}
\end{array}\right)
$$

Here $m_{D}=\frac{1}{\sqrt{2}} Y_{\nu} v$, with $v$ the vacuum expectation value of the SM Higgs boson. Assuming $\mu_{X} \ll m_{D} \ll M_{R}$, the diagonalization of $\mathcal{M}$ leads to an effective Majorana mass matrix for the active (light) neutrinos [37],

$$
m_{\nu} \simeq m_{D}^{T} M_{R}^{T^{-1}} \mu_{X} M_{R}^{-1} m_{D},
$$

whereas the remaining 6 sterile states have masses approximately given by $M_{\nu} \simeq M_{R}$. 
In what follows, and without loss of generality, we work in a basis where $M_{R}$ is a diagonal matrix (as are the charged lepton Yukawa couplings). $Y_{\nu}$ can be written using a modified Casas-Ibarra parametrisation [38] (thus automatically complying with light neutrino data),

$$
Y_{\nu}=\frac{\sqrt{2}}{v} V^{\dagger} \sqrt{\hat{M}} R \sqrt{\hat{m}_{\nu}} U_{\mathrm{PMNS}}^{\dagger},
$$

where $\sqrt{\hat{m}_{\nu}}$ is a diagonal matrix containing the square roots of the three eigenvalues of $m_{\nu}$ (cf. eq. (4.3)); likewise $\sqrt{\hat{M}}$ is a (diagonal) matrix with the square roots of the eigenvalues of $M=M_{R} \mu_{X}^{-1} M_{R}^{T} . V$ diagonalizes $M$ as $V M V^{T}=\hat{M}$, and $R$ is a $3 \times 3$ complex orthogonal matrix, parametrized by 3 complex angles, encoding the remaining degrees of freedom.

The distinctive feature of the ISS is that the additional $\mu_{X}$ parameter allows to accommodate the smallness of the active neutrino masses $m_{\nu}$ for a low seesaw scale, but with natural Yukawa couplings $\left(Y_{\nu} \sim \mathcal{O}(1)\right)$. As a consequence, one can have sizeable mixings between the active neutrinos and the additional sterile states. This is in contrast to the canonical type-I seesaw, where $\mathcal{O}(1)$ Yukawa couplings require $M_{R} \sim 10^{15} \mathrm{GeV}$, thus leading to truly negligible active-sterile mixings.

The nine neutrino mass eigenstates enter the leptonic charged current through their left-handed component (see eq. (1.6), with $i=1, \ldots, 9, j=1, \ldots, 3)$. The unitary leptonic mixing matrix $U_{\nu}$ is now defined as $U_{\nu}^{T} \mathcal{M} U_{\nu}=\operatorname{diag}\left(m_{i}\right)$. Notice however that only the rectangular $3 \times 9$ sub-matrix (first three columns of $U_{\nu}$ ) appears in eq. (1.6) due to the gauge-singlet nature of $\nu_{R}$ and $X$.

In the ISS limit $\left(\mu_{X} \ll m_{D} \ll M_{R}\right)$, and following [39], one can expand the neutrino mass matrix in powers of $\epsilon \equiv m_{D} M_{R}^{-1}$, block-diagonalizing it at leading order in $\epsilon$, thus easily obtaining $U_{\nu}$, and the relevant active-sterile neutrino mixing angle.

\subsection{Numerical evaluation of $\Delta r_{K}$ in the inverse seesaw model}

We numerically evaluate the contributions to $R_{K}$ in the framework of the ISS and address the two scenarios discussed before, which can be translated into ranges for the (random) entries of the $M_{R}$ matrix: scenario $(A)\left(m_{\nu_{s}}<m_{P}\right)-M_{R i} \in[0.1,200] \mathrm{MeV}$; scenario (B) $\left(m_{\nu_{s}}>m_{P}\right)-M_{R i} \in\left[1,10^{6}\right] \mathrm{GeV}$. The entries of $\mu_{X}$ have also been randomly varied in the $[0.01 \mathrm{eV}, 1 \mathrm{MeV}]$ range for both cases.

The adapted Casas-Ibarra parametrisation for $Y_{\nu}$, eq. (4.4), ensures that neutrino oscillation data is satisfied (we use the best-fit values of the global analysis of [24], and set the CP violating phases of $U_{\text {PMNS }}$ to zero). The $R$ matrix angles are taken to be real (thus no contributions to lepton electric dipole moments are expected), and randomly varied in the range $\theta_{i} \in[0,2 \pi]$. Although we do not discuss it here, we have verified that similar $\Delta r_{K}$ contributions are found when considering the more general complex $R$ matrix case.

In figures 1, we collect our results for $\Delta r_{K}$ in scenarios (A) - left panel - and (B) - right panel, as a function of $\tilde{\eta}$, which parametrizes the departure from unitarity of the active neutrino mixing sub-matrix $\tilde{U}_{\mathrm{PMNS}}, \tilde{\eta}=1-\left|\operatorname{Det}\left(\tilde{U}_{\mathrm{PMNS}}\right)\right|$. Although the cosmological constraints are not always satisfied, we stress that all points displayed comply with the different experimental and laboratory bounds discussed before. For the case of scenario (A), one can have very large contributions to $R_{K}$, which can even reach values $\Delta r_{K} \sim \mathcal{O}(1)$ 
Scenario (A)

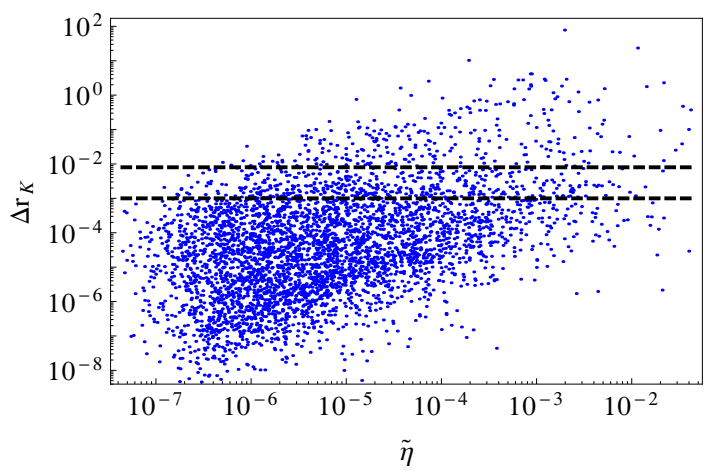

Scenario (B)

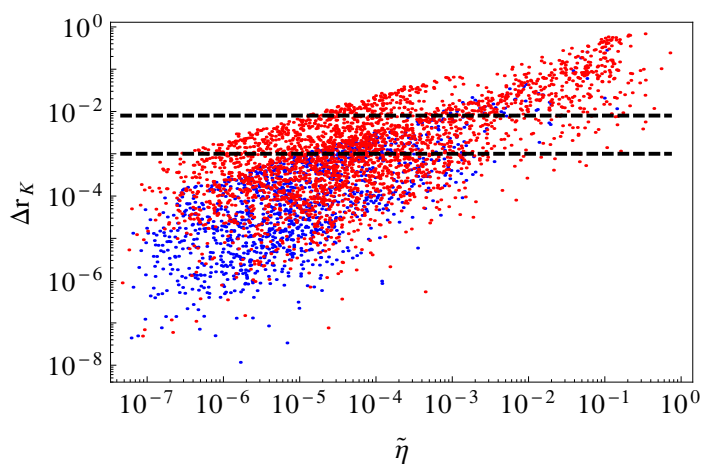

Figure 1. Contributions to $\Delta r_{K}$ in the inverse seesaw as a function of $\tilde{\eta}=1-\left|\operatorname{Det}\left(\tilde{U}_{\mathrm{PMNS}}\right)\right|$ : scenarios A (left) and B (right). The upper (lower) dashed line denotes the current experimental limit (expected sensitivity). On the right panel, red points denote cases where $Y_{\nu} \gtrsim 10^{-2}$. All points comply with experimental and laboratory constraints. Points in (B) are also in agreement with cosmological bounds, while those in (A) require considering a non-standard cosmology.

(in some specific cases we find $\Delta r_{K}$ as large as $\sim 100$ ). The hierarchy of the sterile neutrino spectrum in case (A) is such that one can indeed have a significant amount of LFU violation, while still avoiding non-unitarity bounds. Although this scenario would in principle allow to produce sterile neutrinos in light meson decays, the smallness of the associated $Y_{\nu}$ $\left(\lesssim \mathcal{O}\left(10^{-4}\right)\right)$, together with the loop function suppression $\left(G_{\gamma}\right)$, precludes the observation of LFV processes, even those with very good associated experimental sensitivity, as is the case of $\mu \rightarrow e \gamma$. The strong constraints from CMB and X-rays would exclude scenario (A); in order to render it viable, one would require a non-standard cosmology.

Despite the fact that in case (B) the hierarchy of the sterile states is such that nonunitarity bounds become very stringent (since the sterile neutrinos are not kinematically viable meson decay final states), sizeable LFU violation is also possible, with deviations from the SM predictions again as large as $\Delta r_{K} \sim \mathcal{O}(1)$. Contrary to case (A), whose results could also arise in other frameworks with light sterile neutrinos, the large deviations in (B) typically occur when all the singlet states are considerably heavier than the decaying meson, and reflect specific features of the ISS. As can be inferred from eq. (4.3), in the inverse seesaw framework, one has $m_{\nu} \sim\left(Y_{\nu} v / M_{R}\right)^{2} \mu_{X}$; hence, for "low" (when compared to, for instance, the type I seesaw scale) $M_{R}$, light neutrino data can still be accommodated with large Yukawa couplings, $Y_{\nu} \sim$ few $\times 10^{-1}$. As a consequence, large active-sterile mixings can occur, thus leading to an enhancement of $R_{K}$. Even if in this case one cannot produce sterile states in meson decays, the large $Y_{\nu}$ open the possibility of having larger contributions to LFV observables so that, for example, $\mathrm{BR}(\mu \rightarrow e \gamma)$ can be within MEG reach in this case.

Although we do not explicitly display it here, the prospects for $\Delta r_{\pi}$ are similar: in the same framework, one could have $\Delta r_{\pi} \sim \mathcal{O}\left(\Delta r_{K}\right)$, and thus $\Delta r_{\pi} \sim \mathcal{O}(1)$ in both scenarios. Depending on the singlet spectrum, these observables can also be strongly correlated: if all the sterile states are either lighter than the pion (as it is the case of scenario (A)) or then heavier than the kaon, one finds $\Delta r_{\pi} \approx \Delta r_{K}$. The latter possibilities are a feature of the 
ISS mechanism (not possible in the unconstrained MSSM, for example) and are expected to be present in other low-scale seesaw models that allow for large active-sterile mixing angles.

\section{Concluding remarks}

The existence of sterile neutrinos can potentially lead to a significant violation of lepton flavour universality at tree-level in light meson decays. As shown in this study, provided that the active-sterile mixings are sufficiently large, the modified $W \ell \nu$ interaction can lead to large contributions to lepton flavour universality observables, with measurable deviations from the standard model expectations, well within experimental sensitivity. This mechanism might take place in a number of frameworks, the exact contributions for a given observable being model-dependent.

As an illustrative (numerical) example, we have evaluated the contributions to $R_{K}$ in the inverse seesaw extension of the SM - a truly minimal extension of the SM - , for distinct hierarchies of the sterile states. In particular, we have studied the impact of nonunitarity in a low mass regime for the additional singlets, an inverse seesaw mass regime considerably lower than what had been previously addressed [23, 26]. Recent studies [40] have proposed a search of the monochromatic peak in the next generation of high intensity experiments, yielding both the mass and mixing angles for sterile neutrinos with masses in the range $3 \mathrm{MeV} \lesssim m_{\nu_{s}} \lesssim 414 \mathrm{MeV}$.

Our analysis reveals that very large deviations from the SM predictions can be found $\left(\Delta r_{K} \sim \mathcal{O}(1)\right)$ - or even larger, well within reach of the NA62 experiment at CERN. This is in clear contrast with other models of new physics (for example unconstrained SUSY models, where one typically has $\left.\Delta r_{K} \lesssim \mathcal{O}\left(10^{-3}\right)\right)$. We further notice that these large deviations are a generic and non fine-tuned feature of this model. It is worth emphasising that, in view of the potentially large new contributions to these observables, such an analysis of LFU violation in light meson decays actually allows to set bounds on the amount of unitarity violation (parametrized by $\eta$ ).

Interestingly, in this framework, both $\Delta r_{K}$ and $\Delta r_{\pi}$ are strongly correlated in the case where all the sterile states are lighter than the pion or heavier than the kaon.

The impact of this mechanism is not restricted to light meson decays: there are currently some hints of lepton flavour universality violation in heavy mesons, with deviations already found in observables such as $\Gamma\left(B^{-} \rightarrow \tau \nu\right) / \Gamma\left(\bar{B}^{0} \rightarrow \pi^{+} \ell^{-} \nu\right)$. We expect significant contributions to $B$-meson observables [41], which have very promising experimental perspectives.

\section{Acknowledgments}

We are grateful to Damir Becirevic, Jorge de Blas and Jean-Pierre Leroy for many useful and enlightening discussions. We are also thankful to Robert Shrock for valuable exchanges. This work has been partly done under the ANR project CPV-LFV-LHC NT09-508531. The authors acknowledge partial support from the European Union FP7 ITN INVISIBLES (Marie Curie Actions, PITN- GA-2011- 289442). 
Open Access. This article is distributed under the terms of the Creative Commons Attribution License which permits any use, distribution and reproduction in any medium, provided the original author(s) and source are credited.

\section{References}

[1] V. Cirigliano and I. Rosell, Two-loop effective theory analysis of $\pi(K) \rightarrow e \bar{\nu}_{e}[\gamma]$ branching ratios, Phys. Rev. Lett. 99 (2007) 231801 [arXiv:0707.3439] [INSPIRE].

[2] M. Finkemeier, Radiative corrections to $\pi_{l 2}$ and $K_{l 2}$ decays, Phys. Lett. B 387 (1996) 391 [hep-ph/9505434] [INSPIRE].

[3] NA48/2 and NA62 collaborations, E. Goudzovski, Kaon programme at CERN: recent results, PoS (EPS-HEP2011) 181 [arXiv:1111.2818] [INSPIRE].

[4] G. Czapek et al., Branching ratio for the rare pion decay into positron and neutrino, Phys. Rev. Lett. 70 (1993) 17 [INSPIRE].

[5] NA48/2 and NA62 collaborations, E. Goudzovski, Kaon experiments at CERN: recent results and prospects, arXiv:1208.2885 [INSPIRE].

[6] D. Pocanic et al., New studies of allowed pion and muon decays, arXiv:1210.5025 [INSPIRE].

[7] C. Malbrunot et al., Measurement of the pion branching ratio at TRIUMF, AIP Conf. Proc. 1441 (2012) 564 [INSPIRE].

[8] A. Celis, M. Jung, X.-Q. Li and A. Pich, Sensitivity to charged scalars in $B \rightarrow D^{(*)} \tau \nu_{\tau}$ and $B \rightarrow \tau \nu_{\tau}$ decays, JHEP 01 (2013) 054 [arXiv: 1210.8443] [INSPIRE].

[9] W.-S. Hou, Enhanced charged Higgs boson effects in $B^{-} \rightarrow \tau \bar{\nu}, \mu \bar{\nu}$ and $b \rightarrow \tau \overline{n u}+X$, Phys. Rev. D 48 (1993) 2342 [inSPIRE].

[10] A. Masiero, P. Paradisi and R. Petronzio, Probing new physics through $\mu$-e universality in $K \rightarrow l \nu$, Phys. Rev. D 74 (2006) 011701 [hep-ph/0511289] [INSPIRE].

[11] A. Masiero, P. Paradisi and R. Petronzio, Anatomy and phenomenology of the lepton flavor universality in SUSY theories, JHEP 11 (2008) 042 [arXiv:0807.4721] [INSPIRE].

[12] J. Ellis, S. Lola and M. Raidal, Supersymmetric grand unification and lepton universality in $K \rightarrow l \nu$ decays, Nucl. Phys. B 812 (2009) 128 [arXiv: 0809.5211] [InSPIRE].

[13] J. Girrbach and U. Nierste, $\Gamma(K \rightarrow e \nu) / \Gamma(K \rightarrow \mu \nu)$ in the minimal supersymmetric standard model, arXiv:1202.4906 [INSPIRE].

[14] R. Fonseca, J. Romao and A. Teixeira, Revisiting the $\Gamma(K \rightarrow e \nu) / \Gamma(K \rightarrow \mu \nu)$ ratio in supersymmetric unified models, Eur. Phys. J. C 72 (2012) 2228 [arXiv:1205.1411] [INSPIRE].

[15] A. Kusenko, Sterile neutrinos: the dark side of the light fermions, Phys. Rept. 481 (2009) 1 [arXiv: 0906.2968] [INSPIRE].

[16] J. Schechter and J. Valle, Neutrino masses in $\mathrm{SU}(2) \times \mathrm{U}(1)$ theories, Phys. Rev. D 22 (1980) 2227 [inSPIRE].

[17] M. Gronau, C.N. Leung and J.L. Rosner, Extending limits on neutral heavy leptons, Phys. Rev. D 29 (1984) 2539 [inSPIRE].

[18] R. Shrock, New tests for and bounds on, neutrino masses and lepton mixing, Phys. Lett. B 96 (1980) 159 [INSPIRE]. 
[19] R.E. Shrock, General theory of weak leptonic and semileptonic decays. 1. Leptonic pseudoscalar meson decays, with associated tests for and bounds on, neutrino masses and lepton mixing, Phys. Rev. D 24 (1981) 1232 [INSPIRE].

[20] T. Asaka, S. Blanchet and M. Shaposhnikov, The $\nu M S M$, dark matter and neutrino masses, Phys. Lett. B 631 (2005) 151 [hep-ph/0503065] [INSPIRE].

[21] A. Ibarra, E. Molinaro and S. Petcov, TeV scale see-saw mechanisms of neutrino mass generation, the Majorana nature of the heavy singlet neutrinos and $(\beta \beta)_{0 \nu}$-decay, JHEP 09 (2010) 108 [arXiv: 1007.2378] [INSPIRE].

[22] R. Mohapatra and J. Valle, Neutrino mass and baryon number nonconservation in superstring models, Phys. Rev. D 34 (1986) 1642 [INSPIRE].

[23] M. Malinsky, T. Ohlsson and H. Zhang, Non-unitarity effects in a realistic low-scale seesaw model, Phys. Rev. D 79 (2009) 073009 [arXiv:0903.1961] [INSPIRE].

[24] D. Forero, M. Tortola and J. Valle, Global status of neutrino oscillation parameters after Neutrino-2012, Phys. Rev. D 86 (2012) 073012 [arXiv:1205.4018] [INSPIRE].

[25] A. Atre, T. Han, S. Pascoli and B. Zhang, The search for heavy Majorana neutrinos, JHEP 05 (2009) 030 [arXiv:0901.3589] [INSPIRE].

[26] S. Antusch, J.P. Baumann and E. Fernandez-Martinez, Non-standard neutrino interactions with matter from physics beyond the standard model, Nucl. Phys. B $\mathbf{8 1 0}$ (2009) 369 [arXiv: 0807.1003] [INSPIRE].

[27] A. Abada, D. Das, A. Vicente and C. Weiland, Enhancing lepton flavour violation in the supersymmetric inverse seesaw beyond the dipole contribution, JHEP 09 (2012) 015 [arXiv: 1206.6497] [INSPIRE].

[28] MEG collaboration, J. Adam et al., New limit on the lepton-flavour violating decay $\mu^{+} \rightarrow e^{+} \gamma$, Phys. Rev. Lett. 107 (2011) 171801 [arXiv:1107.5547] [InSPIRE].

[29] R. Alonso, M. Dhen, M. Gavela and T. Hambye, Muon conversion to electron in nuclei in type-I seesaw models, JHEP 01 (2013) 118 [arXiv:1209.2679] [INSPIRE].

[30] A. Ilakovac and A. Pilaftsis, Flavor violating charged lepton decays in seesaw-type models, Nucl. Phys. B 437 (1995) 491 [hep-ph/9403398] [InSPIRE].

[31] F. Deppisch and J. Valle, Enhanced lepton flavor violation in the supersymmetric inverse seesaw model, Phys. Rev. D 72 (2005) 036001 [hep-ph/0406040] [InSPIRE].

[32] Particle Data Group collaboration, J. Beringer et al., Review of particle physics (RPP), Phys. Rev. D 86 (2012) 010001 [inSPIRE].

[33] P. Bhupal Dev, R. Franceschini and R. Mohapatra, Bounds on TeV seesaw models from LHC Higgs data, Phys. Rev. D 86 (2012) 093010 [arXiv:1207.2756] [INSPIRE].

[34] F. del Aguila, J. de Blas and M. Pérez-Victoria, Effects of new leptons in electroweak precision data, Phys. Rev. D 78 (2008) 013010 [arXiv: 0803.4008] [InSPIRE].

[35] A.Y. Smirnov and R. Zukanovich Funchal, Sterile neutrinos: direct mixing effects versus induced mass matrix of active neutrinos, Phys. Rev. D 74 (2006) 013001 [hep-ph/0603009] [INSPIRE].

[36] G. Gelmini, E. Osoba, S. Palomares-Ruiz and S. Pascoli, MeV sterile neutrinos in low reheating temperature cosmological scenarios, JCAP 10 (2008) 029 [arXiv:0803.2735] [INSPIRE]. 
[37] M. Gonzalez-Garcia and J. Valle, Fast decaying neutrinos and observable flavor violation in a new class of Majoron models, Phys. Lett. B 216 (1989) 360 [INSPIRE].

[38] J. Casas and A. Ibarra, Oscillating neutrinos and $\mu \rightarrow e, \gamma$, Nucl. Phys. B 618 (2001) 171 [hep-ph/0103065] [INSPIRE].

[39] D. Forero, S. Morisi, M. Tortola and J. Valle, Lepton flavor violation and non-unitary lepton mixing in low-scale type-I seesaw, JHEP 09 (2011) 142 [arXiv:1107.6009] [INSPIRE].

[40] L. Lello and D. Boyanovsky, Searching for sterile neutrinos from $\pi$ and $K$ decays, arXiv: 1208.5559 [INSPIRE].

[41] A. Abada, A.M. Teixiera, A. Vicente and C. Weiland, work in preparation. 